\title{
FUNDAMENTAL SOLUTION OF THE WAVE EQUATION IN CASE OF A CURVED SPACE-TIME
}

\author{
(Presented by H. Keres)
}

By means of the perturbation expansion scheme with respect to a small parameter $\varepsilon$, the forward fundamental solution $G^{+}$of the general scalar second-order linear hyperbolic differential equation is considered. In the first approximation, the regular part of $G^{+}$is derived in the form of a closed integral expression. In the particular case of a scalar field in a gravitational background field of a point source it is shown that the first-order regular part of $G^{+}$is determined by the energy-momentum vector of the source only.

\section{Introduction}

In this paper we deal with the wave equation which is a general secondorder, linear, hyperbolic differential equation in 4 independent variables. Such an equation can be written in coordinate invariant form as

$$
L_{x} u(x):=g^{i k}(x) u_{; i ; k}+A^{i}(x) u_{, i}+C(x) u=B(x),
$$

where $g^{i k}$ are the contravariant components of the metric tensor of a pseudo-Riemannian space $V^{4}$ of signature $(+-\ldots+)$ and «, , and «;» denote, respectively, the partial derivative and the covariant derivative with respect to the pseudo-Riemannian connection; Latin indices will run from 1 to 4 . The coefficients $g^{i k}, A^{i}$ and $C$ are assumed to be of class $C^{\infty}$.

Of great importance in mathematical and physical applications are the fundamental solutions $G^{ \pm}$(Green's functions) of Eq. (1): The general theory of fundamental solutions is well known (see for example $\left.\left[{ }^{1,2}\right]\right)$; however, their explicit computation is difficult. Until now, the exact fundamental solution of Eq. (1) is explicitly known for some particular cases of curved space-time only. One of the possibilities to treat the general case is the approximate determination of $G^{ \pm}$. For example, the regular part $V^{ \pm}$(tail term) of $G^{ \pm}$can be evaluated in a weak-field approximation as a power series in the square of the geodesic distance $\sigma$ or as a Fourier integral (cf. $[3,4]$ ). However, these forms are cumbersome for the physical application.

In the present paper the geometrical quantities are assumed to depend on a parameter $\varepsilon$, marking the order of deviation of the metric $g^{i k}$ from the Minkowski metric, and are expanded up to the first order in $\varepsilon$, so that the results are formally Lorentz-covariant. This perturbation approach is based on $\left[{ }^{3}\right]$. We avoid the need for expansion in $\sigma$ of the tail term by solving an integral equation for $V^{+}$(see Eq: (8)), which can be solved by successive approximation. According to this integral equation, we obtain the first-order tail term in the form of a closed integral expression, the main properties of which are summarized in Section 5.

Our conventions are: the Ricci tensor $R_{i k}$ is defined as in $\left[{ }^{5}\right] ; g(x):=$ 
$=\operatorname{det}\left(g_{i k}(x)\right)$; the expression $F=0(f)$ is taken to mean that $F / f$ is bounded in the limit, which is stated or implicit from the context; if a geometrical object depends on two points $x$ and $y$ (or $z$ and $y$ ), then the partial differentiation will be denoted by comma for the point $x$ (or $z$ ) only, e. g.,

$$
\partial f(x, y) / \partial x^{i} \equiv f_{, i}(x, y) .
$$

In order to complete the construction of the $G^{ \pm}(x, y)$, we are restricted to a causal domain $\Omega \subseteq V^{4}$, (see $\left.\left[{ }^{2}\right]\right)$.

\section{The fundamental solution}

Consider the general scalar second-order linear differential equation in its covariant form (1) on a causal domain $\Omega$ of a pseudo-Riemannian space-time manifold $V^{4}$ endowed with a given normalhyperbolic $C^{\infty}$ metric $\mathrm{g}^{i k}$. The fundamental solution $G(x, y)$ for this equation satisfies

$$
L_{x} G(x, y)=\delta^{4}(x, y),
$$

where $\delta^{4}(x, y)$ is the Dirac delta distribution, with $\left(\delta^{4}(x, y), \Phi(x)\right):=$ $=\Phi(y)$ for all $\Phi(x) \in C_{0}^{\infty}(\Omega)$.

As in the flat space-time case, there are two fundamental solutions that are particularly important: $G^{+}(x, y)$ and $G^{-}(x, y)$. These are the forward and backward fundamental solutions of (1), respectively.

An object that will be of fundamental importance in the following is $\sigma(x, y)$, the world function of the points $x$ and $y\left[{ }^{6}\right]$. This is a biscalar at $x$ and $y$, numerically equal to a half of the square of the geodesic distance between the points $x$ and $y$, and is positive for time-like intervals and negative for space-like intervals. It has the defining equations

$$
g^{i k}(x) \sigma_{, i}(x, y) \sigma_{, h}(x, y)=2 \sigma(x, y), \sigma(x, y)=\sigma(y, x), \lim _{x \rightarrow y} \sigma=0 .
$$

We shall also be concerned with the distributions $\delta^{ \pm}(\sigma(x, y))$, defined by

$$
\left(\delta^{ \pm}(\sigma(x, y)), \Phi(x)\right):=\int_{C^{ \pm}(y)} \Phi(x) \mu_{\sigma}(x) \text { for all } \Phi(x) \in C_{0}^{\infty}(\Omega),
$$

where $\mu_{\sigma}(x)$ is a Leray form such that

$$
d \sigma(x, y) \wedge \mu_{\sigma}(x)=\mu(x),
$$

$\mu(x)$ is an invariant volume element and the regions of integrations $C^{+}(y)$ and $C^{-}(y)$ are the future null semi-cone and the past null semi-cone, respectively. $\left(C^{+}(y)\right.$ is the set of all points $x \in \Omega$ that can be reached along future-directed null geodesics from $y ; C^{-}(y)$ is defined similarly).

In general, the integral (3) can be evaluated by means of a partition of unity subordinated to a covering of $\Omega$ by open coordinate neighbourhoods in each of which

$$
\mu(x)=\sqrt{-g(x)} d x^{1} \wedge \ldots \wedge d x^{4} .
$$

The relevant properties of the distributions $\delta^{ \pm}(\sigma)$ are given in $\left[{ }^{2}\right]$.

It can be shown that the fundamental solutions $G^{ \pm}$are of the form (see for example $\left.\left[{ }^{2}\right]\right)$ :

$$
G^{ \pm}(x, y)=\frac{1}{4 \pi}\left(W(x, y) \delta^{ \pm}(\sigma(x, y))+V^{ \pm}(x, y)\right) .
$$

It consists of singular parts $W \delta^{ \pm}(\sigma)$ which are the measures, supported 
on $C^{ \pm}(y)$, and of regular parts $V^{ \pm}$, which are the functions; the functions $V \pm(x, y)$ have their support contained in the closures of the sets $D^{ \pm}(y)$ which denote the respective interiors of the future and past pointing characteristic conoids $C^{ \pm}(y)$. The function $W(x, y)$ in Eq. (5) can be expressed as follows:

$$
W(x, y)=\mathrm{Q}^{1 / 2} \exp \left\{-\frac{1}{2} \int_{0}^{1} A_{k}(z(\lambda)) \frac{d z^{k}(\lambda)}{d \lambda} d \lambda\right\},
$$

where $\lambda$ is an affine parameter along the geodesic between $y$ and $x$, with $z(0)=y$ and $z(1)=x$; Q is a biscalar, the scalarized Van Vleck determinant defined by

$$
\varrho(x, y):=(g(x) g(y))^{-1 / 2}\left|\operatorname{det}\left(\partial^{2} \sigma / \partial x^{i} \partial y^{k}\right)\right| .
$$

The regular part $V^{+}(x, y)$ called the tail term, is in $C^{\infty}\left(J^{+}(y)\right) \quad(J \pm(y)$ are the closures of the sets $\left.D^{ \pm}(y)\right)$ and satisfies the integral equation

$$
\begin{gathered}
V^{+}(x, y)+\frac{1}{4 \pi} \int_{C^{+}(y) \cap J^{-}(x)} V^{+}(x, z) L_{z} W(z, y) \mu_{\sigma}(z)= \\
=-\frac{1}{4 \pi} \int_{C^{+}(y) \cap c^{-}(x)} W(x, z) L_{z} W(z, y) \omega(z),
\end{gathered}
$$

where $\omega(z)$ is the 2 -form such that

$$
d \sigma(z, x) \wedge d \sigma(z, y) \wedge \omega(z)=\mu(z) .
$$

Here all differential operators act at $z$.

The integrand on the right-hand side of (8) becomes singular if $x$ tends to a point $x^{*} \in C^{+}(y)$. It can be shown that this integral then tends to

$$
V^{+}\left(x^{*}, y\right)=-\frac{1}{2} W\left(x^{*}, y\right) \int_{0}^{1}\left(L_{z} W(z(\lambda), y) / W(z(\lambda), y)\right) d \lambda
$$

where $\lambda$ is an affine parameter along the geodesic between $y$ and $x^{*}$.

We shall discuss only $V^{+}$, as the corresponding results deduced from $V^{-}$can then be derived by reversing the time orientation on $\Omega$.

\section{A perturbational approach}

We assume the metric $g_{i k}$ depending on a parameter $\varepsilon$ varying in the vicinity of zero and marking the order of deviation of the metric $g_{i k}$ from the Minkowski metric $\eta_{i k}=\operatorname{diag}(1,-1,-1,-1)$ :

$$
\begin{aligned}
& g_{i k}(x)=\eta_{i k}+\varepsilon \gamma_{i k}(x)+0\left(\varepsilon^{2}\right), \\
& g^{i k}(x)=\eta^{i k}-\varepsilon \gamma^{i k}(x)+0\left(\varepsilon^{2}\right) .
\end{aligned}
$$

The coefficients $A^{i}$ and $C$ are assumed to be of the first order in $\varepsilon$, i. e.,

$$
A^{i}=\underset{1}{\varepsilon} A^{i}+0\left(\varepsilon^{2}\right), \quad C=\underset{1}{\varepsilon} C+0\left(\varepsilon^{2}\right) .
$$

Then we suppose that the world function $\sigma(x, y)$ and the other geometrical quantities can also be expanded in the parameter $\varepsilon$. Coefficients in the power series in $\varepsilon$ can be considered as tensors on a Minkowski space-time. Consequently, we make the ansatz 


$$
\sigma(x, y)=\sigma_{0}(x, y)+\varepsilon \sigma_{1}(x, y)+0\left(\varepsilon^{2}\right),
$$

$\sigma_{0}(x, y)$ being the world function of the flat space-time:

$$
\sigma_{0}(x, y)=\frac{1}{2} \eta_{i k} q^{i} q^{k},
$$

where

$$
q^{i}:=x^{i}-y^{i}
$$

In the first order one obtains $\left[{ }^{3}\right]$ :

$$
\sigma_{1}(x, y)=\frac{1}{2} q^{i} q^{k} \int_{0}^{1}\left[\gamma_{i k}\right] d \lambda
$$

where we have used the abbreviation

$$
\text { [f] : }=f\left(\lambda q^{1}+y^{1}, \lambda q^{2}+y^{2}, \lambda q^{3}+y^{3}, \lambda q^{4}+y^{4}\right) .
$$

Concerning the Ricci tensor $R_{i k}(x)$, the ansatz

$$
R_{i k}(x)=\underset{a}{\varepsilon} R_{i k}(x)+0\left(\varepsilon^{2}\right)
$$

leads to

$$
\underset{1}{R_{i k}}(x)=\frac{1}{2}\left\{\gamma_{k, l i}^{l}+\gamma_{i, l k}^{l}-\gamma, i k-\square \gamma_{i k}\right\}
$$

where $\gamma:=\gamma_{i}^{i}, \quad \gamma_{k}^{l}:=\eta^{l i} \gamma_{i k}$ and $\square:=\eta^{i k} \frac{\partial^{2}}{\partial x^{i} \partial x^{k}}$.

Finally, from the conditions (6), (7), (13), and (15) it follows that the function $W(x, y)$ can be expressed as

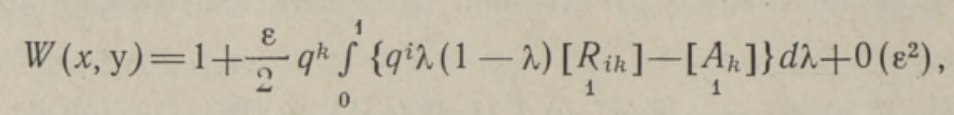

with the notation introduced by Eq. (14).

It should be mentioned that in the case of $A^{i}=C=0$, this expression for $W(x, y)$ was found by R. W. John $\left[{ }^{3}\right]$.

\section{The first-order tail term}

In this section we shall discuss the regular part of the fundamental solution up to the first order in $\varepsilon$

$$
V^{+}(x, y)=\varepsilon V_{1}^{+}(x, y)+0\left(\varepsilon^{2}\right) .
$$

This function distinguishes the general case from the flat space-time case, where $V^{+} \equiv 0$ :

Using the results of the previous section, we can see that in order $\varepsilon$ the integral equation (8) reduces to

$$
\varepsilon V_{1}^{+}(x, y)=-\frac{1}{4 \pi} \int_{C^{+}(y) \cap c^{-}(x)} L_{z} W(z, y) \omega(z)+0\left(\varepsilon^{2}\right),
$$

when $x \in D^{+}(y)$ : Therefore, using the expression (16) and the identities

$$
\underset{1, l}{R_{i, l}}=\frac{1}{2} R_{1}, i \text { with } \underset{1}{R}:=R_{1}^{l},
$$

we arrive, after integration by parts, to the solution 


$$
\begin{aligned}
& V_{1}^{+}(x, y)=-\frac{1}{8 \pi} \int_{S}\left\{\dot { q } ^ { k } \int _ { 0 } ^ { 1 } \lambda ^ { 2 } d \lambda \left(\dot{q}^{i} \lambda(1-\lambda)\left[\begin{array}{c}
\square R_{i k}-\frac{1}{3} R_{1}, i k \\
1
\end{array}\right]+\right.\right. \\
& \left.\left.+\left[-\underset{1}{A_{k}+A_{1}^{i}, i k}\right]\right)+\underset{1}{2} C_{1}+\frac{1}{3} R_{1}-A_{1}^{k}\right\} \omega_{0}(z) \text {, }
\end{aligned}
$$

$x \in D^{+}(y)$, where $\stackrel{*}{*}^{k}:=z^{k}-y^{k}, S:=C_{0}^{+}(y) \cap C_{0}^{-}(x) ; \omega_{0}(z)$ and $C_{0}^{ \pm}(y)$ denote the 2 -form $\omega(z)$ and the future and past pointing characteristic conoids $C^{ \pm}(y)$ of the flat zeroth order space-time, respectively. This is the representation of the tail term $V^{+}$up to the first order in $\varepsilon$.

All the following equations are to be evaluated in flat space-time, in which geometrical quantities should be denoted by index 0 . For the sake of clarity, we omit it in all unambiguous cases.

We shall discuss now some of the main properties of the solution (17).

From Eqs. (9) and (16) it follows that the function $V_{1}^{+}(x, y)$ is continuous on $J^{+}(y)$ and tends to

$$
\begin{aligned}
& V_{1}^{+}(z, y)=-\frac{1}{8} \int_{0}^{1}\left\{4[\underset{1}{C}]+4(\lambda-1)\left(\left[\underset{1}{A_{, i}^{i}}\right]-\lambda \underset{1}{R}\right]+\right. \\
& \left.+\lambda(\lambda-1) \dot{q}^{k}\left(2\left[\square \underset{1}{A_{k}}\right]+\dot{q}^{i} \lambda(\lambda-1)\left[\underset{1}{\square R_{i k}}\right]\right)\right\} d \lambda
\end{aligned}
$$

when $x \rightarrow z \in C^{+}(y)$, in $D^{+}(y)$.

It is worth pointing out that one can derive another, more useful for the purposes of calculation, representation for $V_{1}^{+}(x, y)$. To evaluate the integral in (17), we make a change of variables. Let us take $y$ to be the origin, and denote the coordinates of $z$ by $\left(z^{1}, r, \vartheta, \varphi\right)$, where

$$
\begin{aligned}
& \dot{q}^{2}=r \sin \vartheta \cos \varphi, \\
& \dot{q}^{3}=r \sin \vartheta \sin \varphi, \\
& \dot{q}^{4}=r \cos \vartheta:
\end{aligned}
$$

The corresponding 2 -form $\omega(z)$ on $S$ can be written as

$$
\omega(z)=\left(\sigma_{0}(x, y) /\left(n_{i} q^{i}\right)^{2}\right) \sin \vartheta d \vartheta \wedge d \varphi
$$

with $n_{1}:=1, n_{\alpha}:=-\dot{q}^{\alpha} / r,(\alpha=2,3,4)$.

Let a function $U(x, y)$ be defined by:

$$
U(x, y):=\frac{1}{4 \pi} \int_{S} v(z, y) \omega(z), \text { when } x \in D^{+}(y),
$$

where $v(z, y)$ is a regular function. Then it follows from (20) and (19), by repeated integration by parts, that

$$
\frac{\partial}{\partial x^{k}} U(x, y)=\frac{1}{8 \pi} \int_{S}\left\{v_{, k}+\int_{0}^{1} \lambda^{2} d \lambda\left(\dot{q}^{i}\left[v_{, k i}\right]-\dot{q}_{k}[\square v]\right)\right\} \omega(z)
$$

on $D^{+}(y)$.

Now, we can combine the results of Eqs. (17) and (21) to obtain: 


$$
\begin{aligned}
& V_{1}^{+}(x, y)=\frac{1}{4 \pi} \frac{\partial}{\partial x^{k}}\left\{\int_{S} \dot{q}^{i}\left(\int_{0}^{1} \lambda^{2} d \lambda\left[\underset{1}{G_{i}^{k}}\right]\right) \omega(z)\right\}-
\end{aligned}
$$

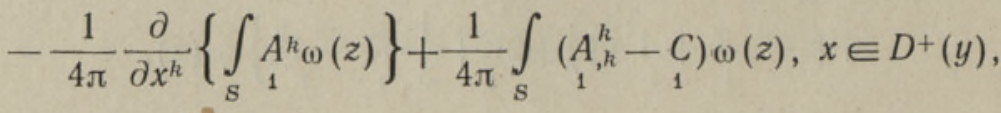

where$$
\underset{1}{G}{ }_{i}^{h}:=\underset{1}{R_{i}^{k}}-\frac{1}{2} \delta_{i}^{h} R
$$

The first integral on the right hand side can be put into the form

$$
\int_{S}\left(q^{i} \int_{0}^{1} \lambda^{2} d \lambda\left[\underset{1}{G_{i}^{k}}\right]\right) \omega(z)=\left(\sigma_{0}(x, y)\right)^{-1} \int_{\Sigma} G_{1}^{k i}(z) d \Sigma_{i}(z) .
$$

Here $\Sigma:=C^{+}(y) \cap J^{-}(x)$ and $d \Sigma_{i}(z)$ denotes the surface element of $\Sigma$ defined as

$$
d \Sigma_{i}(z):=\frac{1}{6} \sqrt{-g(z)} \varepsilon_{j k l i} d z^{j} \wedge d z^{k} \wedge d z^{l},
$$

where $\sqrt{-g(z)} \varepsilon_{j k l i}$ are the covariant components of the discriminant tensor with $\varepsilon_{1234}=-1$.

We can now consider the situation that arises when the supports of the functions $A_{1}^{i}, C_{1}$ and $G_{i k}$ are contained in an interior of a world-tube $\Gamma$ which meets $\Omega$. The Eqs. (22) and (23) imply that a sufficient condition for the vanishing of the tail term $V_{1}^{+}(x, y)$ on a neighbourhood $D$ of $y$ is given by

$$
\Sigma \cap \Gamma=\varnothing, \quad \mathrm{v} x \in D .
$$

On the other hand, if a region of space-time $D^{*} \subset D^{+}(y)$ is such that

$$
C^{+}(y) \cap \Gamma \subset \Sigma \text { with } S \cap \Gamma=\varnothing, \quad \mathrm{V} x \in D^{*},
$$

then the second and third terms on the right hand side of (22) vanish and $V_{1}^{+}(x, y)$ takes the form

$$
V_{1}^{+}(x, y)=-\frac{1}{4 \pi}\left(\sigma_{0}(x, y)\right)^{-2} q^{k} \int_{\Sigma \cap \Gamma} G_{1}^{i}(z) d \Sigma_{i}(z) \text { on } D^{*}
$$

(Note that the 2-surface $S$ is the spatial boundary of $\Sigma$ ). We can apply this to the case when the metric satisfies the Einstein gravitational field equations. These can be written to the first order as

$$
\varepsilon_{1} G_{i k}=8 \pi x T_{i k}+0\left(\varepsilon^{2}\right),
$$

where $x$ is the gravitational constant and the quantities $T_{i k}$ are the covariant components of the energy-momentum tensor of sources on the zeroth order flat space-time. Thus, with the conditions $x T_{k, i}^{i}=0\left(\varepsilon^{2}\right)$ and supp $T^{i k} \subset \Gamma$, we may express the corresponding energy-momentum vector $P$ as

$$
x P^{i}=(\varepsilon / 8 \pi) \int_{\Sigma \cap \Gamma} G_{1}^{i k}(z) d \Sigma_{k}(z)+0\left(\varepsilon^{2}\right),
$$

( $P^{i}$ are the contravariant components of a Lorentz vector on the zeroth order space-time). It then follows from (24) that, for $\mathrm{x} \in D^{*}$,

$$
\varepsilon V_{1}^{+}(x, y)=-2 x\left(\sigma_{0}(x, y)\right)^{-2} q_{i} P^{i} .
$$


Consequently, on the space-time region $D^{*}$ the first order value of the tail term is determined only by the energy-momentum vector $P$ of the gravitational field sources.

Recently the authors of $\left.{ }^{7}\right]$ have obtained analogous results in the particular case of a static gravitational field with $A^{k}=C=0$.

\section{Comparison and conclusions}

The integral equation (8) has been used to derive the formulae (17), (18) and (22) for the first-order tail term $V_{1}^{+}(x, y)$ of the forward fundamental solution. Some properties of $V_{1}^{+}(x, y)$ are given in the following consequences of Eqs. (17), (18) and (22):

1. The tail term $V_{1}^{+}(x, y)$ vanishes for every $x \in \Omega$ and $y \in \Omega$ if and only if the coefficients of the differential equation (1) satisfy the conditions:

$$
\begin{aligned}
& \underset{1}{2 C}-A_{1, i}^{i}+\frac{1}{3} \underset{1}{ } R=0, \\
& \square \underset{1}{A_{i}}-\underset{1, k i}{A^{k}}=0 \text {, } \\
& \square R_{i k}-\frac{1}{6} \eta_{i k} \square R_{1}-\frac{1}{3} R_{1}, i k=0, \quad \forall x \in \Omega .
\end{aligned}
$$

This result follows directly from (17) and (18). It is worth to remark that these conditions in the analytic case were given by P. Günther $\left[{ }^{8}\right]$. 2. Suppose that only in the world-tube $\Gamma$ the conditions (26) are not valid and that the space-time region $D:=\{x: x \in J+(y), \Sigma \cap \Gamma=\varnothing\}$ is not empty. Then the tail term $V_{1}^{+}(x, y)$ vanishes for every $x \in D$ :

3. If the metric satisfies the Einstein equations and if the supports of the sources of gravitational field as well as the supports of $A^{i}$ and $C$ are contained in a world-tube $\Gamma$, then on the space-time region $D^{*}:=$ $=\left\{x: x \in D^{+}(y), C^{+}(y) \cap \Gamma \subset \Sigma, S \cap \Gamma=\varnothing\right\}$ the $V_{1}^{+}(x, y)$ is determined only by the energy-momentum vector of sources.

In particular, if $\Gamma$ can be approximated as a time-like world-line and $y \in \Gamma$, then the sets $D^{*}$ and $D^{+}(y)$ coincide. Hence $V_{1}^{+}(x, y)$ is to the same approximation determined by $(25)$ on $D^{+}(y)$. The singular behaviour of $V_{1}^{+}(x, y)$ on the neighbourhood of the null-cone $C^{+}(y)$ is generated due to the point source approximation. This corollary is in agreement with the result of $\left[{ }^{9}\right]$ where it is shown that the tails of the radial outgoing scalar waves appear in the first order in $\varepsilon$ if and only if the multipole expansion of the gravitational field contains terms describing either a gravitational mass or a linear momentum of the source; the other multipole moments do not contribute to the tail.

The author would like to thank Dr. R. W. John and I. Piir for many valuable discussions.

\section{REFERENCES}

1. De Witt, B. C., Brehme, R. W. Ann. Phys. (N. Y.), 9, № 2, 220-259 (1960).

2. Friedlander, $F$. G. The wave equation on a curved space-time. Cambridge, Cambridge University Press, 1975.

3. John, R. W. Math. Nachr., 60, 109-129 (1974).

4. John, R. W. Ann. phys., 29, Heft 1, 15-26 (1973).

5. Ландау Л. Д., Лифииц Е. М. Теория поля. М., «Наука», 1967. 
6. Synge, J. L. Relativity: the General Theory. Amsterdam, North-Holland Publ. Comp., 1960.

7. Mankin, R., Piir, $I$. ENSV TA Toim. Füüs. Matem., 32, Nr. 2, 157-164 (1983).

8. Günther, P. Arch. Math., 16, 465-475 (1965).

9. Манкин, Р., Пийр И. Препринт FI-29, Тарту, 1974.

Tallinn Polytechnic Institute

Received Jan. 28,1983

\section{R. MANKIN}

\section{LAINEVORRANDI FUNDAMENTAALLAHEND KOVERA AEGRUUMI KORRAL}

Kasutades arendust väikese parameetri $\varepsilon$ astmete järgi, on uuritud hüperboolset tüüpi lineaarse skalaarse teist järku diferentsiaalvõrrandi fundamentaallahendit. Esimeses lähenduses on esitatud selle lahendi regulaarne osa $\left(V^{+}\right)$integraalse avaldisena. On inäidatud, et erijuhul, kui vôrrand kirjeldab skalaarset välja punktallika põhjustatud gravitatsioonivälja foonil, on $V^{+}$esimeses lahenduses täielikult imääratud allika energiaimpulsi vektoriga.

\section{P. МАНКИН}

\section{ФУНДАМЕНТАЛЬНОЕ РЕШЕНИЕ ВОЛНОВОГО УРАВНЕНИЯ В ИСКРИВЛЕННОМ ПРОСТРАНСТВЕ-ВРЕМЕНИ}

$\mathrm{C}$ помощью разложения искомых величин в ряд по степеням малого параметра $\varepsilon$ исследуется фундаментальное решение общего линейного скалярного дифференциального уравнения второго порядка гиперболического типа. Регулярная часть $\left(V^{+}\right)$этого решения в первом приближении представляется в виде замкнутого интегрального выражения.

В случае скалярного поля во внешнем гравитащионном поле точечного источника, в частности, показано, что в первом приближении $V^{+}$определяется полностью вектором энергии-импульса источника. 